\title{
Photon Painter: A robotic device that transfers drawings onto a large surface area using laser beam on photomechanical reaction materials
}

\author{
Paris Xyntarianos-Tsiropinas \\ University of the Aegean \\ Department of Products and Systems \\ Design and Engineering \\ 2 Konstantinoupoleos st. Hermoupolis, \\ Syros, Greece \\ parisxt@aegean.gr
}

\author{
Konstantinos Bailas \\ University of the Aegean \\ Department of Products and Systems \\ Design and Engineering \\ 2 Konstantinoupoleos st. Hermoupolis, \\ Syros, Greece \\ kbail@aegean.gr
}

\author{
Thomas Spyrou \\ University of the Aegean \\ Department of Products and Systems \\ Design and Engineering \\ 2 Konstantinoupoleos st. Hermoupolis, \\ Syros, Greece \\ tsp@aegean.gr
}

\begin{abstract}
The creation of murals - also known as large scale paintings - along with the evolution of street art and graffiti has gained great popularity and in recent years finding applications beyond the field of art. The process of painting murals on walls or other flat surfaces requires time and physical effort. Engineers in collaboration with artists around the globe have developed various provisions and methodologies to optimise this method. Our contribution was the design and assembly of a device called DDArtS (Digitally Drawn Art System). A CNC based machine that proved to be complicated and despite the implementation of various technical improvements, could only help users in the mural creation process by spraying the outlines of the draft sketch on the wall. Having the experience and knowing the problems that arise with the use of the DDArtS system, we have advanced in designing a new device, which is more efficient, requires a shorter setup time and, finally, the user can operate faster and easier. This device consists of (a) a CNC single point projector that uses an ultra violet laser to excite photons on luminescent materials and (b) a robotic base system with a telescopic arm. Design intent was used during the design process of this machine with the use of a parametric CAD system and all the necessary mechanical properties, limitations and constraints to make the final product ready to be build using Additive Manufacturing technology (AM), in a low production line or by a single user.
\end{abstract}

Additive manufacturing. Street art. Photomechanical reaction materials. Robotic. Laser. Murals

\section{INTRODUCTION}

Mural artworks are among the most accessible and recognizable forms of public art. They have been used with a variety of different approaches; they have transferred the values of states or religions in the past, they were used as the voice of the weak while also capturing important historical and social events. Moreover, they often act as a decorative element of the public space, serving the society's needs for an art that pleases through its colours and shapes (Golden et al. 2002, p.20).

The word mural comes from the Latin word mūrālis, which is equivalent to the wall mūr (us) + -âlis -al. In 1390 Cennino Cennini gave the definition of frescoes as "the creative process that takes place on the wall". The art of frescoes is considered to be one of the oldest creative forms of expression, combining a range of techniques and materials that have evolved over the years, connected and shaped its composition: the environment, the artist and the purpose of creation. This creative form of expression, among other things, has been used to inspire and provoke ideas, improve socio-political situations, motivate, embellish and beautify, unite people with their cultural heritage, and even function as a therapeutic agent in human evolution. In any case, mural art conceals a wealth which, despite the form that it takes, gives value to the environment, the artist and the purpose of its creation, having as a catalyst the need for action. 
The reasons above are some that have prompted efforts to build a device that will be able to help artists in the creation of large-scale murals. This effort took shape in the form of the DDArtS system (Xyntarianos-Tsiropinas et al. 2016, 2017, 2018), which has as its basic functional characteristic the creation of a draft sketch on the wall, which the artist can use as a guide for completing a mural. For a review on large-scale murals, existing draft transferring methods with traditional and modern practices, the DDArtS system - its evolution and functionality, the reader can study the three previous related articles presented at EVA in the years 2016, 2017 and 2018.

\section{DDARTS: FUNCTIONAL BUT CUMBERSOME}

After three years of research and development for the construction of the DDArtS and after conducting an experiment that presented important findings about its mode and time of operation (XyntarianosTsiropinas et al. 2018), a decision was taken to create a new and more sophisticated instrument.

Undoubtedly, DDArtS is a functional device, based on simple components and wiring, proven to be able to complete the lines of a draft sketch - either straight or curved, or combining them - in faithful proportions to the digital file and much faster from an inexperienced user or even from a professional artist. However, thinking about the variety of features, materials, tools and technologies available, the DDArtS looks like an old rotary phone compared to modern mobile ones.

Any technological construct always has prospects for evolution. However, DDArtS has some features that make it difficult to further develop:

(i) It takes a lot of time to be assembled and placed on the wall, as well as to transport it (it consists of four stepping motors, the controller, the can and the holder, the cabling system that connects them physically and electronically, a computer and the controlling software).

(ii) It is difficult to be used on dirty surfaces, or generally on other surfaces that are not vertical.

(iii) Whilst it can execute drafts consisting mainly of straight lines with great precision, the programming required for curved lines is demanding and time consuming, while small malfunctions are likely to occur.

(iv) The individual components used for the system, while can be printed on a threedimensional printer, cannot be repaired when hit or broken. There is a high possibility for the components to be hit while the system operates, especially under bad weather conditions or when the system is being used on tall buildings and surfaces.

(v) Last but not least, the use of a spray can for the draft creation leaves a large colour footprint on the wall, such that the painting requires a larger amount of paint to be then used by the artist (especially if the spray colour is a dark one).

For these reasons, it was decided to adopt a new technology and a device capable of overtaking the already existing system in performance. The most basic design need is to quickly replicate the design on the wall with a shorter set-up time and the use of fewer components to create a device as mobile as possible. In addition, since the purpose is to create the draft and not the entire mural, it would be ideal not to use spray cans, but some other graphic means capable of leaving a less intense footprint on the surface.

In the next section of this article, we present the design guidelines and a design concept for this proposed system.

\section{PHOTON PAINTER}

As mentioned in the previous section of the article, two of the main problems in using DDArtS were the portability and use of the spray can, which creates very "intense" outlines, regardless of the colour used.

In an effort for the authors to find solutions, while also performing as the design team behind the Photon Painter, they decided to "take a few steps back" and consider the design problem from scratch. In the search for methods and artefacts that relate to writing on surfaces, most findings concerned different materials that worked by adding colour or material on the wall (paint, marker, spray, chalk, pencil, pen, etc.). They abovementioned methods are primarily additive methods and when used they always have to be supported by a mechanical operator or by the human body itself - just as in the case of DDArtS.

On the other hand, in the search for subtractive methods, despite the fact that there exist cases where the draft or the whole work can be done by scraping (for example in the works of the Portuguese artist Vhills, or as in sharpened frescoes and geometric motifs that are manifestations of traditional culture in different regions of the world), such a system would theoretically need a great amount of energy and time to be operated, while also requiring some kind of mechanical support. This option cannot be supported by the design team, especially since its 
experience with the DDArtS has shown these or similar problems arising many times in the past.

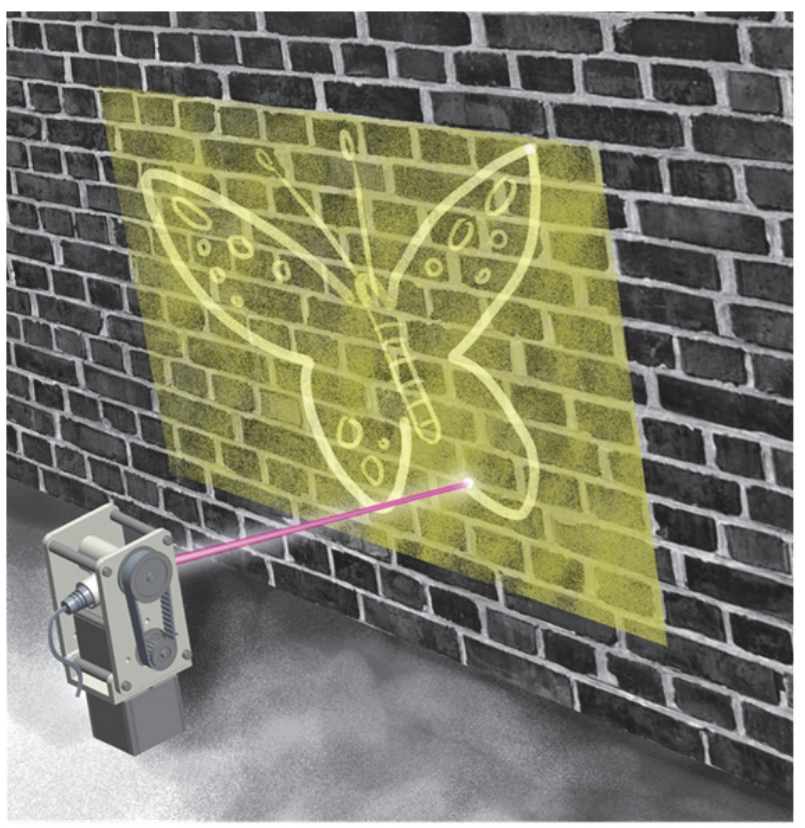

Figure 1: Early concept: The light from the laser beam reacts with the luminescent paint creating the outlines of the desired image.

Thus, with the aim of avoiding the use of such a machine once again, where contact with the wall would be necessary, a basic constraint has emerged. This constraint was very "liberating" for the design team. It was a limitation that helped by directing the team's thought to materials and methods that are capable of "altering" or converting the surface when they come in contact with it. Such methods include light, heat, various liquids, and in some cases even sound. These methods in their vast majority are implemented by making use of chemical reactions. One of the categories of chemically altering materials that excited a strong interest for the team is that of the photosensitive materials (also known as luminescent or phototropic materials). That is, materials that change after contact with specific areas of the light spectrum. In some cases, they change with infrared, while in others with ultraviolet light and radiation.

Considering all of the above, the very basic design guidelines for the system under construction are as follows (without prioritization):

- Be able to create straight and curved lines of a draft sketch on a wall or other surfaces.

- It can operate in small (at least 1 square meter) and large surfaces (up to 100 square meters or more).

- It can work on surfaces that are sloping or rough.
- Allow battery operation if no other power supply is available.

- It can operate under bad weather conditions (air, rain, etc.).

Regarding the technical characteristics of the system, the system should be:

- As light as possible for easier transport.

- As small as possible for better storage and transportation.

- Easy to assemble if it is made up of different parts.

- Easy to interconnect with electronic or electrical sources.

- Durable.

- User-friendly and environmentally friendly.

There are additional guidelines for the laser, the photosensitive paint, the selection and use of specific materials and electrical equipment, but at this stage of the research many things may change. For this reason, these more sophisticated specifications have not yet been settled.
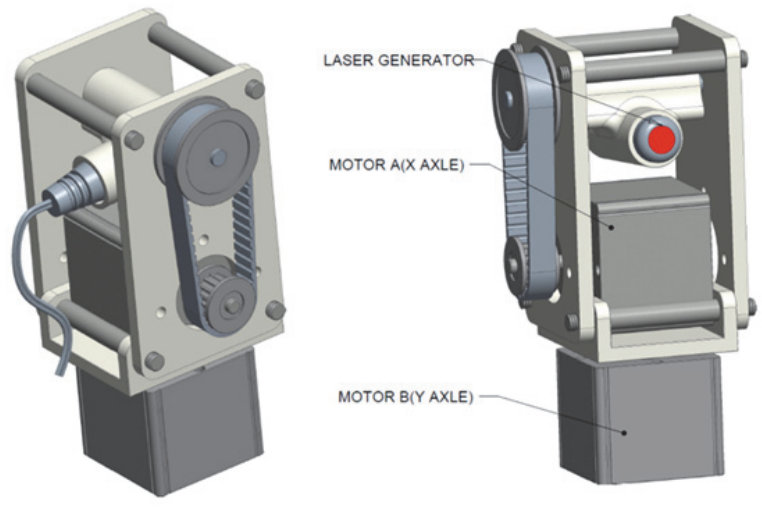

Figure 2: Rendered concept image of the Photon Painter.

\subsection{Using additive manufacturing to create a prototype}

Additive manufacturing (AM) technology, also known as 3D printing, has become more realistic and popular than ever over the last 12 years (Wei et al, 2015). The wide variety of materials that $A M$ uses and the benefits in making complex geometries using low cost materials and machines made it popular in the field of low and mass production in industry ( $\mathrm{Ngo}$ et al. 2018). Many researchers and simple users are combining Computer Aided Design (CAD) systems and low cost Printed Circuit Boards (PCB) such as Arduino, raspberry, etc. to produce parts and assemblies which are then manufactured using AM machines and implement an idea on an integrated basis with full functionality. 
The Photon Painter was designed using a parametric CAD system that enabled us to include all the necessary design information. All of the important parameters and constraints were defined through Design Intent and manufactured using AM technology. The structure of the device was made using an FDM (Fused Deposition Modelling) AM machine to produce the appropriate parts needed. Every single part was then embedded in an assembly with all the electronics parts needed for the device to become operational. The basic function of the Photon Painter lies in the use of a laser beam located on a mobile platform which has two degrees of freedom (Figure 3 ) and two more on a special telescopic arm that are all driven using servo mechanisms in a special moving wheel base (Figure 4).


Figure 3: Laser Generator moves along X, Y axes through servo step motors.
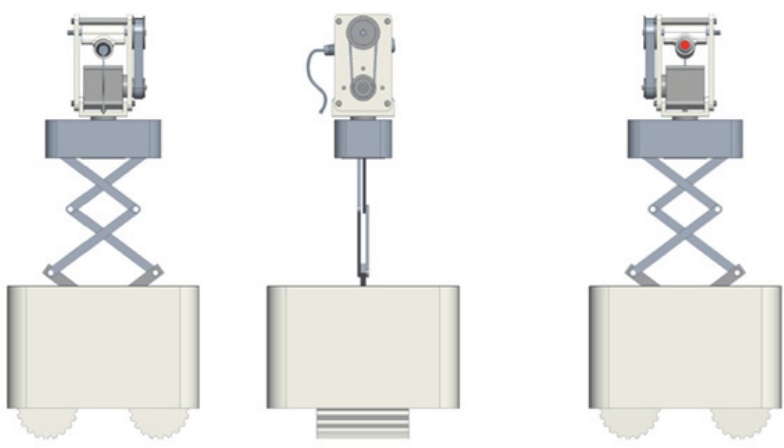

Figure 4: Moving along the $Y$ direction by using a base with a telescopic arm.

The Laser generator uses an ultra violet laser beam to excite photons on the paint area that was painted first with a luminescent material.

Luminescent materials are substances, which convert an incident energy input into the emission of electromagnetic waves in the ultraviolet (UV), visible or infrared regions of the spectrum, over and above that due to blackbody emission (Edgar 2007).

Under a great deal of experiments conducted at the Integrated Industrial Design Lab (INDEL) of the Department of Product and Systems Design Engineering at the University of the Aegean we found that clear coat paint, mixed in some phosphorescent pigment powder gave us the ability to have transparent paint with luminescent properties but further investigation needs to be conducted to find the right formula with the optimum goal to use it on daylight.

The main idea of the device was the laser to shoot at the wall and react with a special photopolymer paint that changes colour and draws intricate and detailed designs (Figure 5). The automation of the device is being controlled from an Arduino that is running an open source motion controller firmware, which is easy to find on the Internet. The laser generator lies on a base that is been controlled by two stepper motors that control the motion on the $X$ and $Y$ ( 2 degrees of freedom) direction. Two more stepper motors control a table that moves along the $X$ axle and a telescopic arm inside it that moves along the $Z$ axle and is responsible for lifting the entire device. This versatility is essential, especially if the laser generator needs to be used on walls with great height. The table that is responsible for the lifting of the device is optional and it is used on the overall construction because we needed to control the distance that the laser generator must have from the wall so that a reactive photochemical reaction can be produced. Further investigation is needed to determine the efficacy of the telescopic arm and how it will operate with the automation of the rest components of the device. The relations between the distances $\mathrm{X}, \mathrm{Y}, \mathrm{Z}$ parameters also need to be determined (Figure 4). These are the distances between the wall and the device through the three directions in space and make a table in which the optional distances for optimum results can be calculated and set according to desired painting sizes.

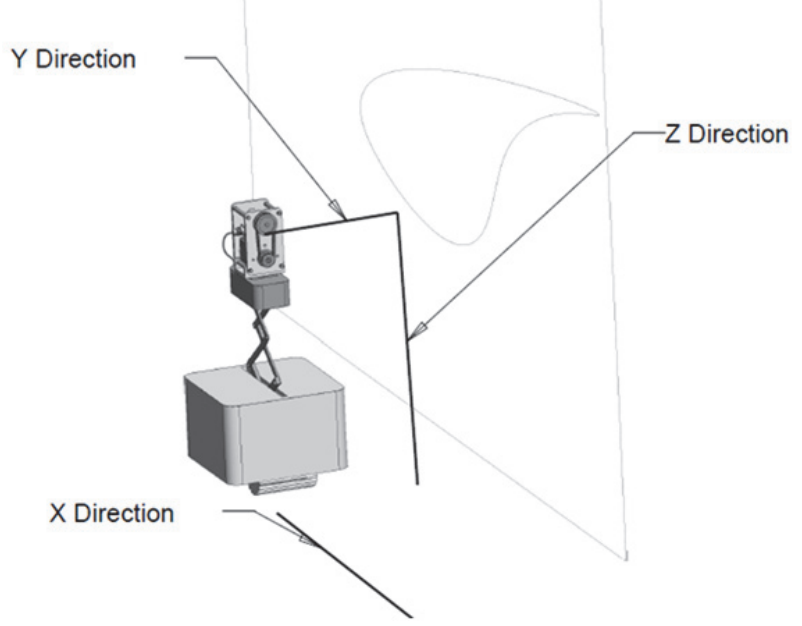

Figure 5: The lower part will be able to move along the $X$ direction, thus allowing the device to operate on all three dimensions. 


\section{CONCLUSIONS}

While a prototype of the Photon Painter has already been made, research into its evolution is still underway. Also, some of the phototropic materials that have been used have not yet produced the results sought by the research team. That is why this article does not include photographic evidence from the laboratory research.

The most important factor throughout this research project was "simplicity" in the machine's construction and operation. For that reason, the design of the entire device was based on the use of Additive Manufacturing technology, which is now widespread with machines that can be accessed by different companies or simple users around the world that make low cost and effective prototypes.

The concept we proposed consists of an assembly with AM parts which control a light source, such as the laser beam proposed, and projects the desired pattern or draft outlines on the wall. A simple robotic arm with two degrees of freedom was used for the movement of the device. The use of the robotic arm is necessary for the position of the laser source to be adjusted in accordance to the size of both the projected image and the area of the wall that will be utilised. There is another purpose in using the robotic arm; it works as an assistive factor for the low power laser beam used in the present phase. In the near future, the device's base will be replaced with a more stable one, which, of course, will have to operate effectively together with the laser beam for the draft sketch to be produced. Our research focuses in the use of a fixed base but always under the condition that the problem of the projection size onto the wall will be solved. This largely depends on the strength of the laser, which, on the other hand, cannot be a very powerful one because it will significantly increase the manufacturing cost of the machine.

\section{REFERENCES}

Arduino Connection (2019) GitHub. https://github.com/synthetos/TinyG/wiki/Connecting -TinyG\#set-up-coolterm (retrieved 13 March 2019).

Edgar, A. (2007) Luminescent Materials. Springer Handbook of Electronic and Photonic Materials, 983-996.

Gao, W., Zhang, Y., Ramanujan, D., Ramani, K., Chen, Y., Williams, C. B., ... and Zavattieri, P. D. (2015) The status, challenges, and future of additive manufacturing in engineering. ComputerAided Design, 69, 65-89.

Golden, J., Rice, R., and Kinney, M. Y., (2002), Philadelphia murals and the stories they tell, Temple University Press, Philadelphia.

Ngo, T. D., Kashani, A., Imbalzano, G., Nguyen, K. T. and Hui, D. (2018). Additive manufacturing (3D printing): A review of materials, methods, applications and challenges. Composites Part B: Engineering, 143, 172-196.

Xyntarianos-Tsiropinas, P., Bailas, K. and Spyrou, T. (2017) The Process of Transferring a Draft Sketch from Small to Large Scale in order to Create Street Artworks: A Case Study Supported by the 'DDArtS. Electronic Visualisation and the Arts (EVA 2017).

Xyntarianos-Tsiropinas, P., Bailas, K. and Spyrou, T. (2017) Using design to support the creation of street art: a three-phase experiment supported by the DDArtS system. Electronic Visualisation and the Arts (EVA 2018).

Xyntarianos-Tsiropinas, P. and Spyrou, T. (2016) DDArtS: Towards designing digitally enhanced street art tools, Electronic Visualisation and the Arts (EVA 2016). 